\title{
DESIGN OF A NEW MAIN INJECTOR CAVITY FOR THE FERMILAB PROTON DRIVER ERA*
}

\author{
V. Wu, A. Chen, Z. Qian, D. W. Wildman, FNAL, Batavia, IL 60510, U.S.A.
}

\begin{abstract}
In the design report of the Fermilab Proton Driver [1], the Main Injector (MI) needs to be upgraded to a $2 \mathrm{MW}$ machine. For the Main Injector radiofrequency (rf) upgrade, $R \& D$ efforts are launched to design and build a new rf system. This paper presents the new cavity design study for the rf system. The cavity is simulated with the design code Mafia [2].
\end{abstract}

\section{INTRODUCTION}

The Fermilab Main Injector is a multi-purpose high energy synchrotron that provides beams for antiproton production, the Tevatron collider and the fixed target experiments. During the collider operations, the Main Injector serves as injector for the Tevatron with an acceleration cycle of 8 to $150 \mathrm{GeV}$ (kinetic energy). For the antiproton production and the fixed target experiments, the acceleration cycles are from 8 to $120 \mathrm{GeV}$. In the baseline design of the Main Injector, it can accelerate $3 \times 10^{13}$ protons per $120 \mathrm{GeV}$ cycle in 1.867 seconds. This corresponds to $0.3 \mathrm{MW}$ of average beam power.

A high average power and tunable energy proton facility has been considered an attractive facility in providing exciting physics opportunities such as neutrino physics at Fermilab after the Tevatron collider program. Extensive upgrade to the existing accelerator complex is required for the new proton facility. The Booster (injector to Main Injector) needs to be replaced by a new machine, called the Proton Driver. There are two Proton Driver design choices, namely, an $8 \mathrm{GeV}$ rapid cycling synchrotron and an $8 \mathrm{GeV}$ superconducting linac. The Main Injector intensity will be increased by a factor of five (over the baseline design value) with a reduced $120 \mathrm{GeV}$ cycle time of 1.5 seconds. With the intensity upgrade, Main Injector becomes a $2 \mathrm{MW}$ machine. One of the major upgrades required of the Main Injector is the rf system.

The rf systems of the Main Injector have several kinds of $\mathrm{rf}$ cavities to serve the various functionalities of the machine. The primary system for beam acceleration has eighteen $53 \mathrm{MHz}$ cavities with a harmonic number of 588 . The cavities have a quality factor (Q) of about 5000 and a shunt impedance $\left(\mathrm{R}_{\mathrm{sh}}\right)$ of $520 \mathrm{k} \Omega$ at $53.1 \mathrm{MHz}$. Besides the $53 \mathrm{MHz}$ cavities, there are $2.5 \mathrm{MHz}, 5 \mathrm{MHz}$ and $106 \mathrm{MHz}$ cavities to do bunch coalescing for producing high intensity beam for the Tevatron. The longitudinal damper has broadband cavities as kickers. For the Proton Driver era, the $53 \mathrm{MHz}$ rf system needs to be upgraded to account for the higher beam power and faster acceleration rate.

\footnotetext{
* Operated by Universities Research Association, Inc. for the U.S.

Department of Energy under contract DE-AC02-76CH03000.

"vincentw@fnal.gov
}

\section{RF SYSTEM REQUIREMENTS}

In the Proton Driver era, the Main Injector intensity will be upgraded to $1.5 \times 10^{14}$ protons with a reduced cycle time of 1.5 seconds. The upgraded MI intensity and acceleration rate are beyond the capability of the existing rf system even if the rf system is upgraded to its maximum power amplifier capability and 20 cavities. Hence, an entirely new cavity system is needed. The new rf system will solve the longitudinal beam stability and beam loading (both transient and steady-state) problems at their source, the cavities. The beam loading voltage $\mathrm{V}_{\mathrm{q}}$ induced by the passage of a bunch through $\mathrm{N}$ cavities is proportional to the bunch charge $\mathrm{q}$ times $\mathrm{N}$ and the $\mathrm{R}_{\mathrm{sh}} / \mathrm{Q}$ (shunt impedance over quality factor) ratio of the cavity, i.e., $\mathrm{V}_{\mathrm{q}} \propto \mathrm{qNR}_{\mathrm{sh}} / \mathrm{Q}$. For the Proton Driver, the MI bunch intensity will be increased by a factor of four over the current operating condition. For the new rf system, $\mathrm{NR}_{\text {sh }} / \mathrm{Q}$ is reduced by a factor of four. Presently, Main Injector can accelerate and store stable beam with the existing rf systems and beam loading compensation. Therefore, an upgraded Main Injector running at four times the current intensity with the new rf system will be stable.

The requirements for the proposed new cavity system are summarized in Table 1. For the new cavity system, the

Table 1: Requirements for the proposed new cavity system for the Proton Driver upgrade

\begin{tabular}{|l|l|}
\hline Frequency $(\mathbf{M H z})$ & $52.813-53.104$ \\
\hline Max. accelerating rate $(\mathbf{G e V} / \mathbf{s})$ & 305 \\
\hline Beam intensity (protons per cycle) & $1.5 \times 10^{14}$ \\
\hline Beam accelerating power $(\mathbf{M W})$ & 7.32 \\
\hline Number of cavities & 18 \\
\hline Cavity $\mathbf{R}_{\text {sh }} / \mathbf{Q}(\mathbf{\Omega})$ & 25 \\
\hline Max. cavity acc. voltage $(\mathbf{k V})$ & 300 \\
\hline
\end{tabular}

beam loading voltage will be kept constant by reducing $\mathrm{R}_{\mathrm{sh}} / \mathrm{Q}$ from $104 \Omega$ to $25 \Omega$. The faster acceleration rate requires the peak accelerating voltage per cavity be increased from $240 \mathrm{kV}$ to $300 \mathrm{kV}$. The design goals of the cavity $\mathrm{Q}$ and $\mathrm{R}_{\mathrm{sh}}$ are 4000 and $100 \mathrm{k} \Omega$, respectively. Basically, the reduction in $\mathrm{R}_{\mathrm{sh}} / \mathrm{Q}$ comes mostly from the shunt impedance. The cavity quality factor remains about the same. The tuning range of the cavity is $52.813 \mathrm{MHz}$ to $53.104 \mathrm{MHz}$. The maximum acceleration rate is assumed to be $305 \mathrm{GeV} / \mathrm{s}$. 


\section{CAVITY SIMULATIONS}

Simulations for the cavity design study are performed with the software Mafia. The Mafia code can simulate a variety of electromagnetic problems in electrostatics, magnetostatics, rf cavity design, etc. Problems can be formulated in two or three dimensions and solved in frequency or time domain. The $53 \mathrm{MHz}$ cavity design is done in two and three dimensions with the eigenmode Mafia solver in frequency domain.

The properties of the unloaded cavity are studied using a two dimensional model. Figure 1 shows a cross section

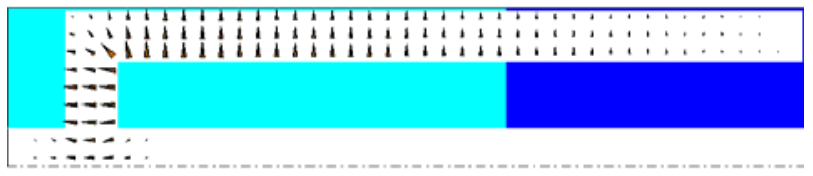

Figure 1: (Color) Mafia model of the cavity with a quarter wave coaxial structure. Only half of the cavity is shown with the cavity axis as the symmetry plane. The electric field shown is the fundamental accelerating mode. The teal color (high electric field region) refers to stainless steel and the blue color refers to copper.

of the bare cavity (without tuner and power amplifier) with the electric field of the fundamental accelerating mode. The cavity is essentially a quarter wave coaxial resonator with a single accelerating gap and a shorted end. It has a total length of about 1.4 meter and a radial extent of about 0.3 meter (from cavity axis). The $\mathrm{R}_{\mathrm{sh}} / \mathrm{Q}$ ratio of a coaxial quarter wave resonator can be adjusted by its characteristic impedance. The material of the cavity body is chosen to be stainless steel to produce high rf losses for shunt impedance reduction. To achieve the desired Q and shunt impedance, the interior surface of the cavity at the high magnetic field region is copper coated. The total amount of copper coating is about 36 degrees of the quarter wave structure. Table 2 shows the properties of the first six TM monopole modes of the cavity spectrum. In the Mafia simulations, $\mathrm{Q}=\omega \mathrm{U}_{\mathrm{T}} / \mathrm{P}$ and $\mathrm{R}_{\mathrm{sh}}=\mathrm{V}^{2} / 2 \mathrm{P}$, where $\omega$ is the angular cavity frequency, $U_{T}$ is the total stored energy, $\mathrm{P}$ is the power loss and $\mathrm{V}$ is the accelerating voltage, are calculated using the field distributions.

Table 2: Properties of the first six TM monopole modes of the cavity spectrum

\begin{tabular}{|l|l|l|l|}
\hline Frequency $(\mathbf{M H z})$ & $\mathbf{Q}$ & $\mathbf{R}_{\text {sh }}(\mathbf{k} \boldsymbol{\Omega})$ & $\mathbf{R}_{\text {sh }} / \mathbf{Q}(\mathbf{\Omega})$ \\
\hline 52.98 & 4000 & 100 & 25 \\
\hline 158.05 & 4000 & 40.5 & 10 \\
\hline 260.29 & 5300 & 45 & 8.4 \\
\hline 357.81 & 6500 & 55 & 8.4 \\
\hline 451.76 & 6700 & 49.3 & 7.4 \\
\hline 547.38 & 7700 & 41 & 5.3 \\
\hline
\end{tabular}

The required cavity frequency tuning range is $291 \mathrm{kHz}$ which is larger than the natural cavity bandwidth of $13 \mathrm{kHz}$. Frequency tuning will be provided by a single ferrite tuner located at the shorted end. The tuner is a copper coaxial resonator filled with 30 aluminum doped yttrium iron garnets with $0.5 \mathrm{~cm}$ garnet separation. It will be perpendicularly biased. The dimensions of the garnets are $32 \mathrm{~cm}$ (outer diameter) $\times 12 \mathrm{~cm}$ (inner diameter) $\times$ $1 \mathrm{~cm}$ (thickness). Tuners with dc bias magnetic field transverse to the rf magnetic field have lower ferrite losses and require less ferrite as compared to tuners with parallel bias. The perpendicular bias field will be provided by a single layer of solenoidal coils around the tuner. For transversely biased tuners, the permeability $\left(\mu_{\mathrm{r}}\right)$ is related to the bias magnetic intensity $\left(\mathrm{H}_{\mathrm{dc}}\right)$ by $\mu_{\mathrm{r}}=1+4 \pi \mathrm{M}_{\mathrm{s}} / \mathrm{H}_{\mathrm{dc}}$, where $4 \pi \mathrm{M}_{\mathrm{s}}$ is the saturation magnetization of ferrite. During acceleration, the bias field will vary from $300 \mathrm{G}$ to $2250 \mathrm{G}$ to produce a change in $\mu_{\mathrm{r}}$ of 2.5 to 1.2 . For all simulations, a permittivity $\left(\varepsilon_{\mathrm{r}}\right)$ of 13.5 is used for the garnets.

The cavity, tuner and power amplifier system is simulated with a three dimensional model in frequency domain to study the frequency tuning capability of the cavity. Figure 2 shows the simulation model with the

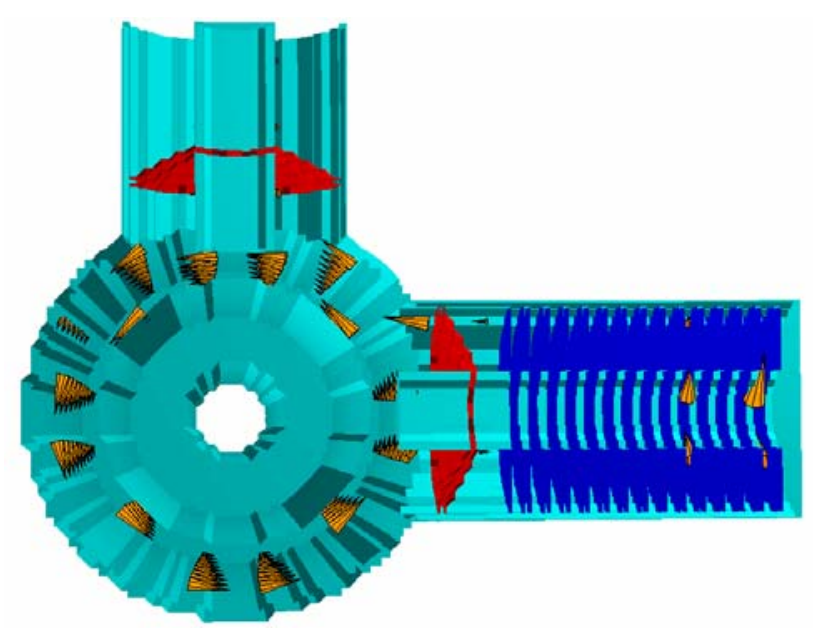

Figure 2: (Color) Three dimensional simulation model showing the transverse cross section of the cavity (on the left with beam pipe hole), tuner (on the right) and the driver (on the top). The magnetic field of the fundamental accelerating mode is also shown.

transverse cross section at the tuner and the driver location. The power amplifier (a tetrode) on the cavity is located 90 degrees azimuthally from the tuner. It is simulated as a coaxial structure with magnetic coupling to the cavity end wall. The ceramic window of the driver is also included. Figure 3 shows the longitudinal cross section of the simulation model with the electric field of the fundamental mode. The tuner is magnetically coupled to the cavity end wall via the tuner center conductor. Tuner coupling is determined by the loop area, which is adjusted by changing the insertion depth of the loop into the cavity. The cavity tuning range depends on the 


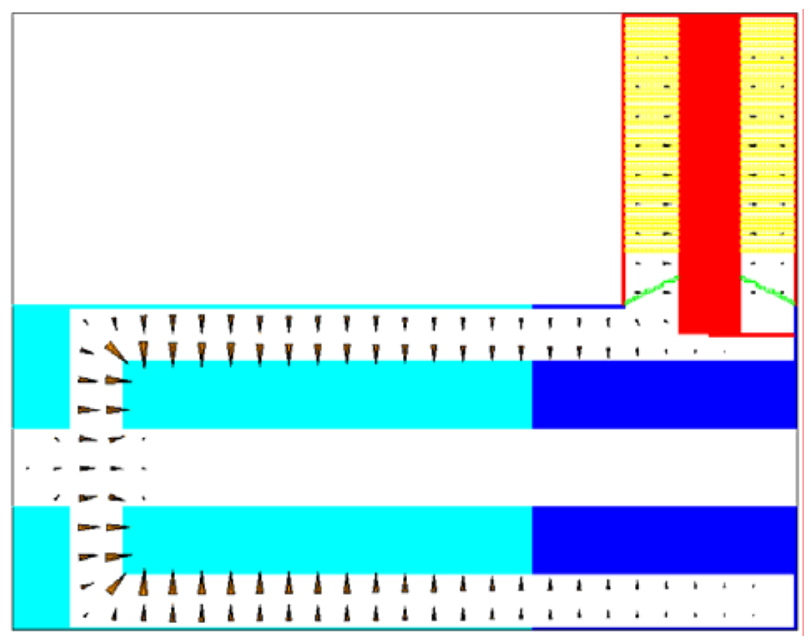

Figure 3: (Color) Longitudinal cross section of the cavity system with the electric field of the fundamental mode shown. The driver located 90 degrees from the tuner is not shown. The ceramic window separating the tuner and the cavity vacuums are also included in the model.

permittivity, the change in $\mu_{\mathrm{r}}$ of the garnets and the amount of tuner coupling to the cavity. The tuning range as a function of tuner coupling to the cavity is studied and the result is shown in Fig. 4. The cavity length is used to tune the center frequency of the cavity. Simulations show that the cavity has ample tuning range. The maximum tuning range is about $1166 \mathrm{kHz}$ which corresponds to the tuner center conductor in contact with the inner conductor of the cavity.

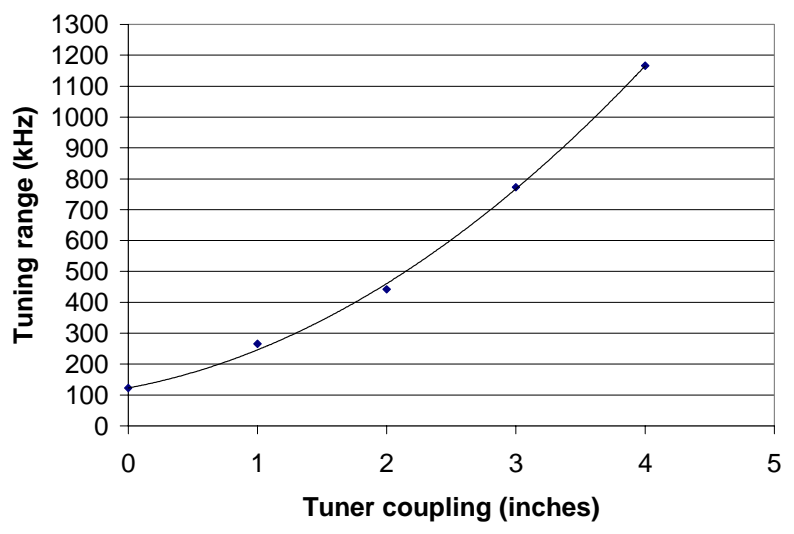

Figure 4: The cavity tuning range is plotted as a function of the tuner coupling (which is the amount of tuner coupling loop insertion into the cavity). Four inches of insertion into the cavity corresponds to the case of the tuner center conductor touching the cavity inner conductor, i.e., maximum coupling to the cavity. Zero inch insertion corresponds to the coupling loop at the outer cavity radius position. The permittivity $\left(\varepsilon_{\mathrm{r}}\right)$ is 13.5 and the change in the permeability $\left(\mu_{\mathrm{r}}\right)$ is from 2.5 to 1.2 .

The cavity and tuner system is also simulated with a different code, called HFSS [3]. HFSS simulations yield the same results as those of the Mafia code.

\section{MECHANICAL DESIGN}

Figure 5 shows the mechanical design of the new cavity. The accelerating gap with rounded corner is shown on the right side of the figure. The vertical coaxial structure on the left is the tuner. The tuner will be air and water cooled. Input jacks on the end walls are water inputs and outputs of the cooling system. The amount of heat load of the cavity (maximum power dissipation in the cavity wall) is about $450 \mathrm{~kW}$. Water cooling of the cavity body is provided by cylindrical sheets of water running along the exterior cavity walls longitudinally. Two ports for higher order mode dampers are included.

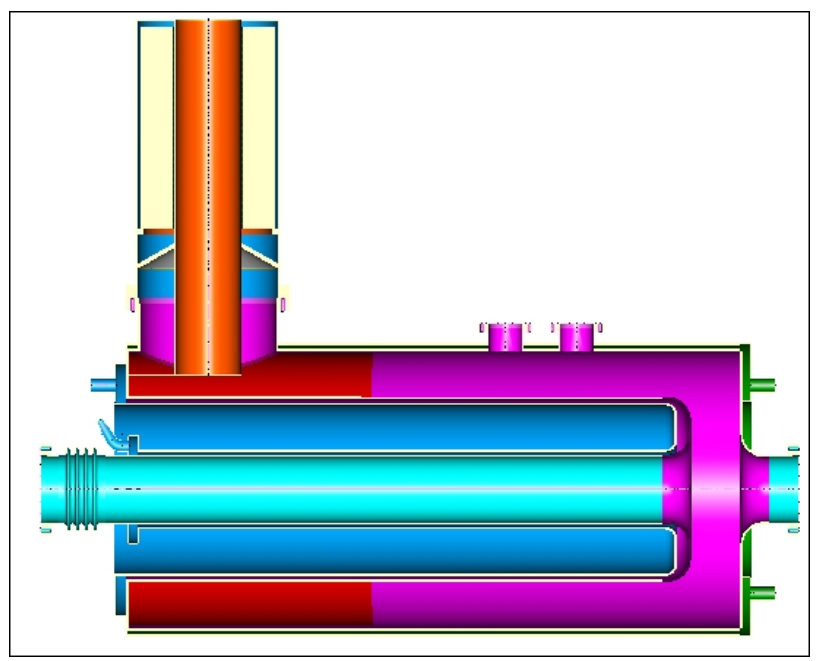

Figure 5: (Color) Mechanical design of the new cavity. The accelerating gap is shown on the right. The tuner is shown on the top left.

\section{CONCLUSION}

A new Main Injector $53 \mathrm{MHz}$ rf cavity has been simulated with the software Mafia for the Proton Driver era. The cavity is designed to accelerate a beam intensity of $1.5 \times 10^{14}$ (per cycle) which is five times the original design intensity of the Main Injector. Simulations show that the new cavity design has satisfactory properties. The mechanical design of the cavity is also presented. The next logical step in this R\&D effort is to build a prototype cavity to test the cavity and the tuner properties. Finally a beam test of the cavity in the Main Injector should be carried out to determine its performance.

\section{REFERENCES}

[1] Proton Driver Study II, FERMILAB-TM-2169, May 2002, edited by G. W. Foster, W. Chou and E. Malamud.

[2] Computer Simulation Technology, MAFIA 4, December 1996.

[3] Ansoft, HFSS v9.0, May 2003. 